\title{
Development and validation of a novel nomogram for predicting the prognosis of patients with resected pancreatic adenocarcinoma
}

\author{
HU REN, CHAO-RUI WU, SADERBIEKE AIMAITI and CHENG-FENG WANG \\ Department of Pancreatic and Gastric Surgery, National Cancer Center/National Clinical Research Center \\ for Cancer/Cancer Hospital, Chinese Academy of Medical Sciences and \\ Peking Union Medical College, Beijing 100021, P.R. China
}

Received October 14, 2019; Accepted March 6, 2020

DOI: $10.3892 / \mathrm{ol} .2020 .11495$

\begin{abstract}
The survival prediction for patients with resected pancreatic adenocarcinoma by using the Tumor-Node-Metastasis (TNM) staging system remains limited. A nomogram is a efficient tool that can be used to predict the outcome of patients with various types of malignancy. The present study aimed to develop and validate a nomogram for patients with resected pancreatic adenocarcinoma. A total of 368 patients (258 in the training set and 110 in the validation set) who underwent pancreatic adenocarcinoma resection at the China National Cancer Center between January 2008 and October 2018 were included in the present study. The nomogram was established according to the results from Cox multivariate analysis, which was validated by discrimination and calibration. The area under the receiver operating characteristic curve (AUC) was determined to assess the accuracy of survival predictions. The results from multivariate analysis in the training set demonstrated that blood transfusion, T-stage, $\mathrm{N}$-stage, tumor grade, capsule invasion, carbohydrate antigen 199, neutrophil percentage and adjuvant therapy were independent prognostic
\end{abstract}

Correspondence to: Professor Cheng-Feng Wang, Department of Pancreatic and Gastric Surgery, National Cancer Center/National Clinical Research Center for Cancer/Cancer Hospital, Chinese Academy of Medical Sciences and Peking Union Medical College, 17 Panjiayuan Nanli, Beijing 100021, P.R. China

E-mail: ywwangchengfeng@163.com

Abbreviations: AJCC, American Joint Committee on Cancer; ASA, American Society of Anesthesiologists; AUC, area under the curve; CA199, carbohydrate antigen 199; CA242, carbohydrate antigen 242; CRP/ALB, c reactive protein/albumin; MLR, monocyte and lymphocyte ratio; NLR, neutrophil and lymphocyte ratio; NP, neutrophil percentage; OS, overall survival; PLR, platelet and lymphocyte ratio; PNI, prognostic nutritional index; SII, systemic inflammatory reaction Index; SIRI, systemic inflammatory response index; TNM, Tumor-Node-Metastasis

Key words: pancreatic adenocarcinoma, nomogram, prognostic factor, survival, Tumor-Node-Metastasis factors for overall survival (OS; all $\mathrm{P}<0.05)$. Subsequently, a nomogram predicting the 1-year, 3-year and 5-year OS rates, with favorable calibration, was established based on the independent prognostic factors. The concordance indices of the nomogram were higher compared with the TNM staging system in both training and validation sets. Furthermore, a clear risk stratification system based on the nomogram was used to classify patients into the three following groups: Low-risk group ( $\leq 168)$, moderate-risk group (168-255) and high-risk group ( $>255)$. The risk stratification system demonstrated an improved ability in predicting the 1-year, 3-year and 5-year OS rates compared with the TNM system (AUC, $0.758,0.709$ and 0.672 vs. AUC, 0.614, 0.604 and 0.568; all $\mathrm{P}<0.05)$. The present study developed and validated a nomogram for patients with resected pancreatic adenocarcinoma by including additional independent prognostic factors, including tumor marker, immune index, surgical information, pathological data and adjuvant therapy. Taken together, the results from the present study indicated an improved performance of the nomogram in predicting the prognosis of patients with resected pancreatic adenocarcinoma compared with the TNM staging system.

\section{Introduction}

Pancreatic adenocarcinoma is the fourth and sixth most common cause of cancer-associated mortality in the United States $(7.27 \%$; 2018) and China $(2.82 \%$; 2015), respectively $(1,2)$. With the annual increase in the number of pancreatic adenocarcinoma-associated mortality cases, it is predicted that this malignancy will become the second leading cause of cancer-associated mortality in the United States by 2030 (3). At present, complete surgical resection remains the only therapeutic strategy for significantly prolonging the survival of patients with pancreatic adenocarcinoma (4). However, since pancreatic adenocarcinoma is diagnosed at late and advanced stages, $<20 \%$ of newly diagnosed patients are eligible for potential curative surgical resection (4,5). Despite recent advancements in chemotherapy and radiotherapy, the 5-year survival rate of patients who underwent curative resection remains poor (12-27\%) (6-8). It is therefore essential to develop an efficient prognostic system to predict the survival of patients with pancreatic adenocarcinoma. 
The American Joint Committee on Cancer (AJCC) Tumor-Node-Metastasis (TNM) staging system (9), which is based on the histological analysis of tumor and metastasis, has been extensively used for the prognostic evaluation of patients with pancreatic adenocarcinoma $(10,11)$. However, the current TNM staging system for pancreatic adenocarcinoma does not include certain clinicopathological factors that may affect the prognosis, including age, sex, tumor grade and carbohydrate antigen 199 (CA199) level (11-13). CA199, as a measure of tumor burden, is a diagnostic and prognostic marker $(14,15)$. Humphris et al (16) reported that normal preoperative CA199 levels identified a good prognosis. Furthermore, previous studies reported that the prognosis of patients varies significantly within the same TNM stage $(10,11)$. It is therefore essential to develop a more efficient predictive model that could be used to predict the survival of patients with pancreatic adenocarcinoma.

Nomograms are multivariate predictive models that have been extensively applied for clinical prognostic evaluation in several types of malignancy (17-20). Unlike the TNM staging system, nomograms are efficient in incorporating additional prognostic factors, allowing therefore a more accurate prediction $(21,22)$. At present, numerous nomograms have been established for predicting the survival of patients with pancreatic adenocarcinoma; however, most of these nomograms used the demographic and pathological data from the United States, where a number of potential prognostic factors, including tumor markers and surgical information, were not included (13,23-27).

The present study aimed to develop and validate a prognostic nomogram for patients with resected pancreatic adenocarcinoma, by including additional prognostic factors based on the clinical database from the China National Cancer Center.

\section{Materials and methods}

Patient dataset and study design. The present study was a retrospective study approved by the Ethics Committee of National Cancer Center/Cancer Hospital, Chinese Academy of Medical Sciences and Peking Union Medical College (Beijing, China). A total of 368 patients with pancreatic adenocarcinoma who underwent curative surgical resection (with an R0 margin) at the China National Cancer Center between January 2008 and October 2018 were included in the present study. The cohort was comprised of $208(56.5 \%)$ male and $160(43.5 \%)$ female patients with a median age of 60 years (age range, $45-72$ years). The study inclusion criteria were as follows: i) Diagnosis of pancreatic adenocarcinoma; ii) patients underwent curative surgical resection with R0 resection, which was determined by no macroscopic or microscopic residual carcinoma; and iii) based on the TNM staging system, the stages of patients were T1-3N+M0 (9). The exclusion criteria were as follows: i) Patients with T4 stage or distant metastasis; ii) patients that underwent palliative surgery; iii) patients who had received preoperative chemotherapy or radiotherapy; iv) patients who had suffered from other malignancies before pancreatic adenocarcinoma and (v) patients who died due to other reasons or unexpected outcomes. Prior to radical pancreaticoduodenectomy or distal pancreatectomy with splenectomy, patients were examined with enhanced MRI and/or CT scanning to confirm the absence of locally unresectable or distant metastases. Generally, there were more people in the training set used to construct the nomogram and fewer people in the validation set. In the present study, the patients were randomly divided into study sets for model training (258 patients) and validation (110 patients) according to a ratio of 7:3 .

All clinicopathological characteristics were collected from the medical record database of the China National Cancer Center and included the following: Age at the time of diagnosis, sex, clinical symptoms (pain, jaundice, digestive symptoms and weight loss), past medical history (diabetes and hypertension) and life style (smoking status and alcohol consumption). The laboratory data included CA199, carbohydrate antigen 242 (CA242), neutrophil percentage (NP), alanine aminotransferase, aspartate aminotransferase, total bilirubin, prognostic nutritional index (PNI), platelet and lymphocyte ratio (PLR), neutrophil and lymphocyte ratio (NLR), monocyte and lymphocyte ratio (MLR), systemic inflammatory reaction index (SII), systemic inflammatory response index (SIRI) and c reactive protein/albumin (CRP/ALB), which were collected 2 weeks prior to radical surgery. The PNI was calculated based on the serum albumin and lymphocyte counts, by using the following equation: PNI $=10 \mathrm{x}$ albumin $(\mathrm{g} / \mathrm{dl})+0.005 \mathrm{x}$ lymphocyte count $\left(/ \mathrm{mm}^{3}\right)$. The SII and SIRI were calculated based on neutrophil, lymphocyte, monocyte and platelet counts, using the following equation: $\mathrm{SII}=$ platelet count $x$ neutrophil count/lymphocyte count; SIRI=neutrophil count $\mathrm{x}$ monocyte count/lymphocyte count. The remaining data were calculated as follows: PLR=platelet count/lymphocyte count; NLR=neutrophil count/lymphocyte count; MLR=monocyte count/lymphocyte count.

The American Society of Anesthesiologists classification (ASA class) and information about blood transfusion, total retrieved lymph nodes, tumor information (location, tumor grade, and lymphovascular, perineural and capsule invasions) and adjuvant therapy were also included in the present study. The tumor stage and nodal involvement in the present study were defined according to the 8th edition TNM staging system (28).

Follow-up analysis. The primary endpoint of the present study was the overall survival (OS), which was measured from the date of radical surgery to the mortality due to pancreatic adenocarcinoma, or to the last follow-up (updated in May 2019). The follow-up procedure was as follows: Once every three months within the first two years following surgery and continual procedures every six months thereafter, in which the postoperative treatment information and survival conditions were recorded. Long-term prognostic data were collected from the patients' clinical records or contact with the patients' relatives via telephone. Patients who died after the surgery (within 1 month following surgery) were excluded from the present study. A total of three patients were lost due to perioperative mortality.

Statistical analysis. X-tile software (v.3.6.1; Yale School of Medicine) (29) was used to identify the optimal cut-off values of the potential prognostic factors, including NP, PNI, PLR, NLR, MLR, SII, SIRI and CRP/ALB. X-tile software can divide marker data into two populations: low and high. All possible 
Table I. Clinicopathological characteristics of patients in the training and validation sets.

\begin{tabular}{|c|c|c|c|c|c|}
\hline \multirow[b]{2}{*}{ Characteristic } & \multicolumn{2}{|c|}{ Training set } & \multicolumn{2}{|c|}{ Validation set } & \multirow[b]{2}{*}{ P-value } \\
\hline & Patient, $\mathrm{n}$ & $\%$ & Patient, $\mathrm{n}$ & $\%$ & \\
\hline Sex & & & & & 0.675 \\
\hline Male & 144 & 55.8 & 64 & 58.2 & \\
\hline Female & 114 & 44.2 & 46 & 41.8 & \\
\hline Age, years & & & & & 0.265 \\
\hline$\leq 60$ & 136 & 52.7 & 51 & 46.4 & \\
\hline$>60$ & 122 & 47.3 & 59 & 53.6 & \\
\hline Symptom & & & & & 0.108 \\
\hline No & 39 & 15.5 & 24 & 22.6 & \\
\hline Yes & 212 & 84.5 & 82 & 77.4 & \\
\hline Pain & & & & & 0.195 \\
\hline No & 104 & 41.6 & 52 & 49.1 & \\
\hline Yes & 146 & 58.4 & 54 & 50.9 & \\
\hline Jaundice & & & & & 0.452 \\
\hline No & 182 & 72.8 & 73 & 68.9 & \\
\hline Yes & 68 & 27.2 & 33 & 31.1 & \\
\hline Digestive symptoms & & & & & 0.023 \\
\hline No & 202 & 80.8 & 74 & 69.8 & \\
\hline Yes & 48 & 19.2 & 32 & 30.2 & \\
\hline Weight loss & & & & & 0.420 \\
\hline No & 152 & 60.6 & 69 & 65.1 & \\
\hline Yes & 99 & 39.4 & 37 & 34.9 & \\
\hline Diabetes & & & & & 0.342 \\
\hline No & 189 & 73.3 & 85 & 78.0 & \\
\hline Yes & 69 & 26.7 & 24 & 22.0 & \\
\hline Hypertension & & & & & 0.131 \\
\hline No & 197 & 76.4 & 75 & 68.8 & \\
\hline Yes & 61 & 23.6 & 34 & 31.2 & \\
\hline Smoke & & & & & 0.231 \\
\hline No & 193 & 76.0 & 77 & 70.0 & \\
\hline Yes & 61 & 24.0 & 33 & 30.0 & \\
\hline Alcohol & & & & & 0.218 \\
\hline No & 204 & 80.3 & 82 & 74.5 & \\
\hline Yes & 50 & 19.7 & 28 & 25.5 & \\
\hline ASA class & & & & & 0.405 \\
\hline$\leq 2$ & 188 & 74.6 & 85 & 78.7 & \\
\hline$>2$ & 64 & 25.4 & 23 & 21.3 & \\
\hline Blood transfusion & & & & & 0.372 \\
\hline No & 155 & 61.5 & 61 & 56.5 & \\
\hline Yes & 97 & 38.5 & 47 & 43.5 & \\
\hline Tumor location & & & & & 0.348 \\
\hline Head and neck & 124 & 48.1 & 47 & 42.7 & \\
\hline Body and tail & 134 & 51.9 & 63 & 57.3 & \\
\hline T stage & & & & & 0.917 \\
\hline $\mathrm{T} 1$ & 33 & 13.1 & 15 & 13.9 & \\
\hline $\mathrm{T} 2$ & 139 & 55.2 & 56 & 56.5 & \\
\hline $\mathrm{T} 3$ & 80 & 31.7 & 35 & 29.6 & \\
\hline N stage & & & & & 0.961 \\
\hline No & 142 & 56.3 & 61 & 53.7 & \\
\hline $\mathrm{N} 1$ & 84 & 33.3 & 35 & 34.3 & \\
\hline $\mathrm{N} 2$ & 26 & 10.4 & 10 & 12.0 & \\
\hline
\end{tabular}


Table I. Continued.

\begin{tabular}{|c|c|c|c|c|c|}
\hline \multirow[b]{2}{*}{ Characteristic } & \multicolumn{2}{|c|}{ Training set } & \multicolumn{2}{|c|}{ Validation set } & \multirow[b]{2}{*}{ P-value } \\
\hline & Patient, $\mathrm{n}$ & $\%$ & Patient, $\mathrm{n}$ & $\%$ & \\
\hline Lymph nodes, $\mathrm{n}$ & & & & & 0.798 \\
\hline$\leq 6$ & 69 & 25.7 & 31 & 27.4 & \\
\hline$>6$ & 171 & 74.3 & 82 & 72.6 & \\
\hline Tumor grade & & & & & 0.473 \\
\hline Poorly & 37 & 14.9 & 17 & 16.4 & \\
\hline Moderately & 174 & 69.9 & 78 & 69.1 & \\
\hline Well & 38 & 15.3 & 11 & 14.5 & \\
\hline $\begin{array}{l}\text { Lymphovascular } \\
\text { invasion }\end{array}$ & & & & & 0.973 \\
\hline No & 174 & 74.0 & 82 & 73.9 & \\
\hline Yes & 61 & 26.0 & 29 & 26.1 & \\
\hline Perineural invasion & & & & & 0.965 \\
\hline No & 95 & 39.7 & 44 & 40.0 & \\
\hline Yes & 144 & 60.3 & 66 & 60.0 & \\
\hline Capsule invasion & & & & & 0.508 \\
\hline No & 70 & 27.7 & 33 & 31.1 & \\
\hline Yes & 183 & 72.3 & 73 & 68.9 & \\
\hline $\mathrm{CEA}, \mathrm{ng} / \mathrm{ml}$ & & & & & 0.370 \\
\hline$\leq 5$ & 158 & 63.7 & 64 & 58.7 & \\
\hline$>5$ & 90 & 36.3 & 45 & 41.3 & \\
\hline $\mathrm{CA} 199, \mathrm{U} / \mathrm{ml}$ & & & & & 0.907 \\
\hline$\leq 37$ & 56 & 22.6 & 24 & 22.0 & \\
\hline$>37$ & 192 & 77.4 & 85 & 78.0 & \\
\hline $\mathrm{CA} 242, \mathrm{IU} / \mathrm{ml}$ & & & & & 0.174 \\
\hline$\leq 20$ & 96 & 38.7 & 34 & 31.2 & \\
\hline$>20$ & 152 & 61.3 & 75 & 68.8 & \\
\hline NP & & & & & 0.686 \\
\hline$\leq 0.700$ & 180 & 72.0 & 80 & 74.1 & \\
\hline$>0.700$ & 70 & 38.0 & 28 & 25.9 & \\
\hline ALT, U/1 & & & & & 0.712 \\
\hline$\leq 40$ & 145 & 63.9 & 66 & 66.0 & \\
\hline$>40$ & 82 & 36.1 & 34 & 34.0 & \\
\hline AST, U/l & & & & & 0.960 \\
\hline$\leq 40$ & 156 & 68.7 & 69 & 69.0 & \\
\hline$>40$ & 71 & 31.3 & 31 & 31.0 & \\
\hline TBIL, $\mu \mathrm{mol} / 1$ & & & & & 0.232 \\
\hline$\leq 17.1$ & 143 & 63.0 & 56 & 56.0 & \\
\hline$>17.1$ & 84 & 37.0 & 44 & 44.0 & \\
\hline PNI & & & & & 0.402 \\
\hline$\leq 52$ & 156 & 68.7 & 64 & 64.0 & \\
\hline$>52$ & 71 & 31.3 & 36 & 36.0 & \\
\hline PLR & & & & & 0.234 \\
\hline$\leq 210.082$ & 222 & 88.8 & 91 & 84.3 & \\
\hline$>210.082$ & 28 & 11.2 & 17 & 15.7 & \\
\hline NLR & & & & & 0.405 \\
\hline$\leq 2.751$ & 162 & 64.8 & 65 & 60.2 & \\
\hline$>2.751$ & 88 & 35.2 & 43 & 39.8 & \\
\hline MLR & & & & & 0.341 \\
\hline$\leq 0.279$ & 163 & 65.2 & 76 & 70.4 & \\
\hline$>0.279$ & 87 & 34.8 & 32 & 29.6 & \\
\hline
\end{tabular}


Table I. Continued.

\begin{tabular}{|c|c|c|c|c|c|}
\hline \multirow[b]{2}{*}{ Characteristic } & \multicolumn{2}{|c|}{ Training set } & \multicolumn{2}{|c|}{ Validation set } & \multirow[b]{2}{*}{ P-value } \\
\hline & Patient, $\mathrm{n}$ & $\%$ & Patient, $\mathrm{n}$ & $\%$ & \\
\hline SII & & & & & 0.770 \\
\hline$\leq 718.312$ & 198 & 79.2 & 87 & 80.6 & \\
\hline$>718.312$ & 52 & 20.8 & 21 & 19.4 & \\
\hline SIRI & & & & & 0.120 \\
\hline$\leq 0.782$ & 110 & 43.0 & 38 & 35.2 & \\
\hline$>0.782$ & 140 & 56.0 & 70 & 64.8 & \\
\hline CRP/ALB & & & & & 0.700 \\
\hline$\leq 0.142 \%$ & 51 & 38.1 & 23 & 41.1 & \\
\hline$>0.142 \%$ & 83 & 61.9 & 33 & 58.9 & \\
\hline Adjuvant therapy & & & & & 0.279 \\
\hline No & 128 & 50.2 & 62 & 56.4 & \\
\hline Yes & 127 & 49.8 & 48 & 43.6 & \\
\hline
\end{tabular}

In the present study, patients who lacked the results of 1-2 factors but had other available factors were also included. ASA class, American Society of Anesthesiologists classification; T, tumor; N, node; CEA, carcinoembryonic antigen; CA199, carbohydrate antigen 199; CA242, carbohydrate antigen 242; NP, neutrophil percentage; ALT, alanine aminotransferase; AST, aspartate aminotransferase; TBIL, total bilirubin; PNI, prognostic nutritional index; PLR, platelet and lymphocyte ratio; NLR, neutrophil and lymphocyte ratio; MLR, monocyte and lymphocyte ratio; SII, systemic inflammatory reaction Index; SIRI, systemic inflammatory response index; CRP/ALB, c reactive protein/albumin.

divisions of the marker data were assessed by a variety of standard statistical tests, including the log-rank test for survival and means tests for associations between other marker data. Then the program selected the optimal division of the data (29). $\chi^{2}$ test was used to compare the categorical characteristics between the two disjoint sets. Cox proportional hazard univariate and multivariate regression analyses were used to evaluate the independent prognostic predictors for OS in the training set.

Multivariate analysis was performed to identify the independent prognostic factors, in order to establish the nomogram by using the rms package within $\mathrm{R}$ project software. Based on the hazard ratio of the corresponding characteristics in the Cox regression model, each independent prognostic factor was scored using the 'nomogram' function within $\mathrm{R}$ project (21). Validations were performed in the validation set using two parameters of the nomogram, discrimination and calibration. The concordance (C)-index was implemented to assess discrimination, whereas calibration curves were generated using bootstrap resampling (1,000 resamples). The area under the receiver operating characteristic curve (AUC) was determined to assess the accuracy of survival predictions. The Kaplan-Meier method was used to analyze the survival curves, and differences were estimated using log-rank test.

Statistical analyses were performed using SPSS software (version 25.0; IBM Corp.), GraphPad Prism software (version 7.00; GraphPad Software Inc.) and R software (version 3.5.2; http://www.r-project.org). Two-tailed $\mathrm{P}<0.05$ was considered to indicate a significant difference.

\section{Results}

Patient characteristics. A total of 368 patients with resected pancreatic adenocarcinoma were randomly divided into the training set (258 patients) and the validation set (110 patients). As presented in Table I, the optimal cut-off values identified using X-tile software were as follows: 0.700 for NP, 52 for PNI, 210.082 for PLR, 2.751 for NLR, 0.279 for MLP, 718.312 for SII, 0.782 for SIRI and $0.142 \%$ for CRP/ALB. Based on these values, patients were subsequently divided into low and high expression groups. The mean follow-up period of the training set was 44.4 months (range, 2-104.9 months), while the 1-year, 3 -year and 5-year OS rates were 72.3, 41.4 and 26.4\%, respectively. The mean follow-up period of the validation set was 40.1 months (range, 1.5-102.8 months), while the 1-year, 3-year and 5-year OS rates were $66.4,38.8$ and $25.6 \%$, respectively. The clinicopathological characteristics of patients from the training and validation sets are presented in Table I. No significant difference was observed between the two groups for each characteristic.

Independent prognostic factors in the training set. The results from univariate and multivariate regression analyses for the training set are presented in Table II. Results from univariate analysis demonstrated that OS was significantly associated with sex, symptom, weight loss, ASA class, blood transfusion, tumor location, $\mathrm{T}$ stage, $\mathrm{N}$ stage, tumor grade, capsule invasion, CA199, CA242, NP, NLR, SII and adjuvant therapy (all $\mathrm{P}<0.05)$. Furthermore, following multivariate analysis, blood transfusion, T stage, $\mathrm{N}$ stage, tumor grade, capsule invasion, CA199, NP and adjuvant therapy were identified as significant independent prognostic factors for patients with resected pancreatic adenocarcinoma (all $\mathrm{P}<0.05$ ).

Development and validation of the nomogram. A nomogram predicting the 1-year, 3-year and 5-year OS rates of patients with pancreatic adenocarcinoma was constructed based on 
Table II. Univariate and multivariate analyses on clinicopathological characteristics of patients in the training set.

\begin{tabular}{|c|c|c|c|c|}
\hline \multirow[b]{2}{*}{ Characteristics } & \multicolumn{2}{|c|}{ Univariate } & \multicolumn{2}{|c|}{ Multivariate } \\
\hline & $\mathrm{HR}(95 \% \mathrm{CI})$ & P-value & HR (95\% CI) & P-value \\
\hline \multicolumn{5}{|l|}{ Sex } \\
\hline Female & Reference & & Reference & \\
\hline Male & $1.370(1.025-1.832)$ & 0.033 & $1.333(0.903-1.966)$ & 0.148 \\
\hline \multicolumn{5}{|l|}{ Symptom } \\
\hline Yes & Reference & & Reference & \\
\hline No & $0.654(0.438-0.978)$ & 0.039 & $0.833(0.489-1.420)$ & 0.501 \\
\hline \multicolumn{5}{|l|}{ Weight loss } \\
\hline Yes & Reference & & Reference & \\
\hline No & $0.734(0.548-0.983)$ & 0.038 & $0.804(0.532-1.215)$ & 0.300 \\
\hline \multicolumn{5}{|l|}{ ASA class } \\
\hline$>2$ & Reference & & Reference & \\
\hline$\leq 2$ & $0.689(0.485-0.978)$ & 0.037 & $1.119(0.686-1.827)$ & 0.652 \\
\hline \multicolumn{5}{|l|}{ Blood transfusion } \\
\hline Yes & Reference & & Reference & \\
\hline No & $0.644(0.469-0.884)$ & 0.007 & $0.594(0.384-0.920)$ & 0.020 \\
\hline \multicolumn{5}{|l|}{ Tumor location } \\
\hline Body and tail & Reference & & Reference & \\
\hline Head and neck & $0.720(0.539-0.961)$ & 0.026 & $0.853(0.522-1.394)$ & 0.525 \\
\hline \multicolumn{5}{|l|}{ T stage } \\
\hline $\mathrm{T} 3$ & Reference & & Reference & \\
\hline $\mathrm{T} 2$ & $0.589(0.431-0.807)$ & 0.001 & $0.513(0.326-0.808)$ & 0.003 \\
\hline $\mathrm{T} 1$ & $0.494(0.300-0.814)$ & 0.006 & $0.451(0.215-0.946)$ & 0.035 \\
\hline \multicolumn{5}{|l|}{$\mathrm{N}$ stage } \\
\hline $\mathrm{N} 2$ & Reference & & Reference & \\
\hline N1 & $0.704(0.425-1.167)$ & 0.174 & $0.592(0.317-1.106)$ & 0.100 \\
\hline No & $0.417(0.254-0.685)$ & 0.001 & $0.400(0.212-0.754)$ & 0.005 \\
\hline \multicolumn{5}{|l|}{ Tumor grade } \\
\hline Well & Reference & & Reference & \\
\hline Moderately & $2.464(1.462-4.155)$ & 0.001 & $2.894(1.383-6.057)$ & 0.005 \\
\hline Poorly & $4.102(2.255-7.460)$ & 0.000 & $3.904(1.671-9.121)$ & 0.002 \\
\hline \multicolumn{5}{|l|}{ Capsule invasion } \\
\hline Yes & Reference & & Reference & \\
\hline No & $0.585(0.405-0.843)$ & 0.004 & $0.496(0.297-0.829)$ & 0.007 \\
\hline \multicolumn{5}{|l|}{ CA199, U/ml } \\
\hline$>37$ & Reference & & Reference & \\
\hline$\leq 37$ & $0.477(0.318-0.717)$ & 0.000 & $0.430(0.230-0.802)$ & 0.008 \\
\hline \multicolumn{5}{|l|}{ CA242, IU/ml } \\
\hline$>20$ & Reference & & Reference & \\
\hline$\leq 20$ & $0.524(0.362-0.758)$ & 0.001 & $0.961(0.573-1.611)$ & 0.880 \\
\hline \multicolumn{5}{|l|}{ NP } \\
\hline$>0.700$ & Reference & & Reference & \\
\hline$\leq 0.700$ & $0.577(0.402-0.827)$ & 0.003 & $0.361(0.198-0.657)$ & 0.001 \\
\hline \multicolumn{5}{|l|}{ NLR } \\
\hline$>2.751$ & Reference & & Reference & \\
\hline$\leq 2.751$ & $0.653(0.475-0.897)$ & 0.008 & $1.301(0.723-2.343)$ & 0.380 \\
\hline \multicolumn{5}{|l|}{ SII } \\
\hline$>718.312$ & Reference & & Reference & \\
\hline$\leq 718.312$ & $0.665(0.464-0.952)$ & 0.026 & 1.097 (0.614-1.960) & 0.754 \\
\hline
\end{tabular}


Table II. Continued.

Univariate
Multivariate

HR $(95 \% \mathrm{CI}) \quad$ P-value
Characteristics

Adjuvant therapy

Yes

No

\author{
Reference
}

1.433 (1.067-1.925)
P-value
Reference

$1.931(1.297-2.875)$

HR, hazard ratio; CI, confidence interval; ASA class, American Society of Anesthesiologists classification; T, tumor; N, node; CA199, carbohydrate antigen 199; CA242, carbohydrate antigen 242; NP, neutrophil percentage; NLR, neutrophil and lymphocyte ratio; SII, systemic inflammatory reaction index.

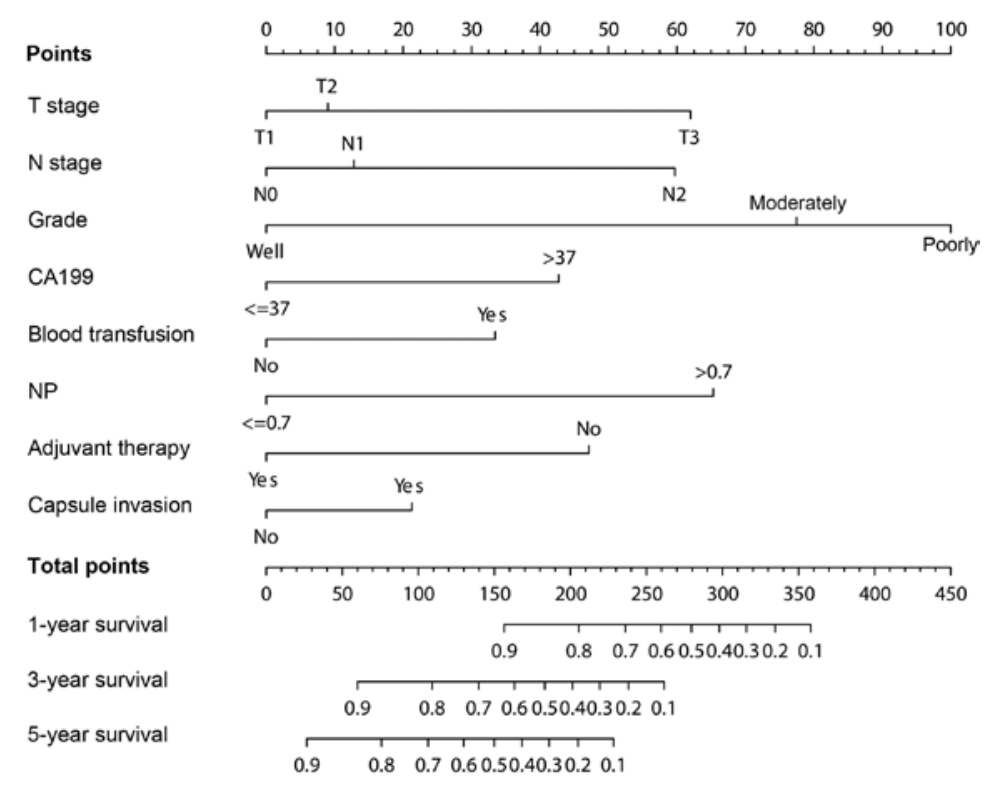

Figure 1. Nomogram predicting the 1-year, 3-year and 5-year overall survival of patients with resected pancreatic adenocarcinoma. T, tumor; N, node; CA199, carbohydrate antigen 199; NP, neutrophil percentage.

the independent prognostic factors identified from the Cox multivariate regression model in the training set (Fig. 1). Each subtype within these indicators was allocated a score according to the 'Points' in the nomogram. Next, the predicted survival probabilities for each patient was calculated using the nomogram. For example, the total points of patients were calculated by summing up the score for each indicator first. Then the right position in the total points axis was found and a perpendicular line drawn to the survival probabilities axis. Finally, the predicted survival probabilities at 1-year, 3-year and 5-year were obtained.

As presented in Table III, the C-indices for the 1-year, 3-year and 5-year OS prediction in the training set were 0.824 [95\% confidential interval (CI), 0.775-0.873], 0.782 (95\% CI, $0.745-0.823$ ) and 0.770 (95\% CI, 0.731-0.810), respectively. The C-indices for the 1-year, 3-year and 5-year OS prediction in the validation set were 0.779 (95\% CI, 0.705-0.853), 0.778 (95\% CI, 0.718-0.838) and 0.766 (95\% CI, 0.709-0.823), respectively. The $\mathrm{C}$-indices of both training and validation sets were higher in the nomogram compared with the TNM staging system. Furthermore, the calibration curves for the probability of 1-year, 3-year and 5-year OS demonstrated a positive association between the predicted and observed values in the validation set (Fig. 2).

Survival analysis according to the risk stratification system based on the nomogram. A clear risk stratification system of OS rates was established using the predicted probabilities obtained from the nomogram. The score for each independent prognostic factor is presented in Table IV. Total scores for each patient was defined as the sum of each score for each indicator in the nomogram. According to the optimal cut-off values of total scores, patients were classified into three groups as follows: Low-risk group $(\leq 168, n=124)$, moderate-risk group $(168-255, n=126)$ and high-risk group $(>255, n=118)$, where each group represented a distinct prognosis. Patients were categorized according to the TNM staging system and the risk stratification system, and the risk stratification system based on the nomogram had the ability to delineate three different prognosis groups $(\mathrm{P}<0.01$; Fig. 3$)$. The AUC values of the risk stratification system for predicting the 1-year, 3-year and 5 -year OS rates were $0.758,0.709$ and 0.672 , respectively, 
Table III. C-indexes for the nomogram and TNM staging system.

\begin{tabular}{|c|c|c|c|c|c|c|}
\hline \multirow[b]{2}{*}{ Characteristics } & \multicolumn{2}{|c|}{ 1-year OS } & \multicolumn{2}{|c|}{ 3-year OS } & \multicolumn{2}{|c|}{ 5-year OS } \\
\hline & C-index & $95 \% \mathrm{CI}$ & C-index & $95 \% \mathrm{CI}$ & C-index & $95 \% \mathrm{CI}$ \\
\hline \multicolumn{7}{|l|}{ Training set } \\
\hline Nomogram & 0.824 & $0.775-0.873$ & 0.782 & $0.742-0.823$ & 0.770 & $0.731-0.810$ \\
\hline TNM system & 0.667 & 0.591-0.742 & 0.648 & $0.589-0.706$ & 0.642 & $0.585-0.699$ \\
\hline \multicolumn{7}{|l|}{ Validation set } \\
\hline Nomogram & 0.779 & $0.705-0.853$ & 0.778 & $0.718-0.838$ & 0.766 & $0.709-0.823$ \\
\hline TNM system & 0.695 & $0.603-0.787$ & 0.672 & $0.595-0.749$ & 0.669 & $0.594-0.744$ \\
\hline
\end{tabular}

C, concordance; TNM, tumor-node-metastasis; OS, overall survival; CI, confidence interval.
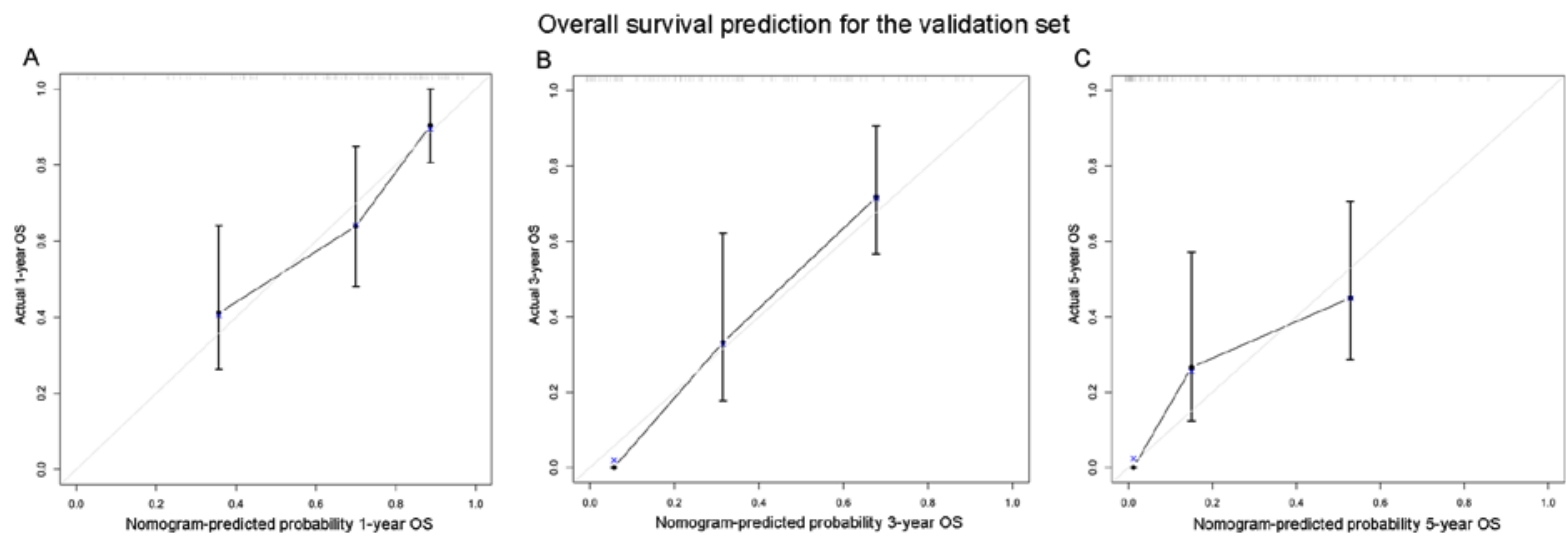

Figure 2. Calibration curves predicting the OS at (A) 1-year, (B) 3-year and (C) 5-year for the validation set. The plots along the $45^{\circ}$ line indicates an appropriate calibration model, in which the predicted probabilities were identical to the actual outcomes. OS, overall survival.

whereas the AUC values for the current TNM staging system are $0.614,0.604$ and 0.568 , respectively (28) (Fig. 4).

\section{Discussion}

The AJCC TNM staging system is currently the most extensively used system to predict the prognosis of several types of cancer, including pancreatic adenocarcinoma (30). Numerous studies suggested that $\mathrm{T}$ and $\mathrm{N}$ stages might not be the only clinical factors that can be used to determine the prognosis of patients with pancreatic adenocarcinoma $(9,13,14,31)$. Since implementing the traditional TNM staging system for patients with resected pancreatic adenocarcinoma is considered as imprecise, it is therefore essential to develop a more accurate survival predictive model $(13,14,31,32)$. In order to address the limitations of the TNM staging system, the present study developed and validated a survival predictive nomogram for patients with resected pancreatic adenocarcinoma, by including additional independent prognostic factors.

Nomogram is a graphical representation of a statistical predictive model, which can predict patients' individualized risk for a specific survival outcome (21). At present, a number of prognostic nomograms have been developed to diagnose several types of cancer, such as non-small-cell lung cancer and gastroenteropancreatic neuroendocrine neoplasms $(21,33,34)$.
The first nomogram for patients with pancreatic adenocarcinoma was established in 2004 by Memorial Sloan-Kettering Cancer Center (MSKCC) (23), which provided more accurate survival predictions compared with the TNM staging system when validated by external patient cohorts $(13,35)$. However, tumor markers, adjuvant therapy and other potential prognostic factors were not considered in the MSKCC study. Furthermore, in the MSKCC nomogram, patients with T1 stage were assigned with higher scores than patients with $\mathrm{T} 2$ and $\mathrm{T} 3$ stages, which is different from clinical data from patients, limiting the popularization of MSKCC nomogram (31). Furthermore, other available nomograms are not specific to patients with resected pancreatic adenocarcinoma $(24,26,33,36)$, whereas the nomogram established in the present study was specifically targeted for patients with resected pancreatic adenocarcinoma. The present nomogram included $\mathrm{T}$ stage, $\mathrm{N}$ stage, tumor grade, capsule invasion, CA199, NP, blood transfusion and adjuvant therapy, which demonstrated an improved performance in predicting the prognosis of patients compared with the TNM staging system. To the best of our knowledge, the present study was the first to describe a nomogram that included capsule invasion, NP and blood transfusion to predict the OS of patients with resected pancreatic adenocarcinoma.

$\mathrm{T}$ and $\mathrm{N}$ stages were included in the present nomogram, as they have previously been considered as key independent 
Table IV. Score of characteristics in the nomogram.

\begin{tabular}{|c|c|}
\hline Characteristic & Score \\
\hline \multicolumn{2}{|c|}{ Blood transfusion } \\
\hline No & 0 \\
\hline Yes & 33.3 \\
\hline \multicolumn{2}{|l|}{ NP } \\
\hline$\leq 0.7$ & 0 \\
\hline$>0.7$ & 65.1 \\
\hline \multicolumn{2}{|l|}{ CA199, U/ml } \\
\hline$\leq 37$ & 0 \\
\hline$>37$ & 42.6 \\
\hline \multicolumn{2}{|l|}{ T stage } \\
\hline $\mathrm{T} 1$ & 0 \\
\hline $\mathrm{T} 2$ & 9 \\
\hline $\mathrm{T} 3$ & 62.1 \\
\hline \multicolumn{2}{|l|}{$\mathrm{N}$ stage } \\
\hline No & 0 \\
\hline N1 & 12.6 \\
\hline $\mathrm{N} 2$ & 59.8 \\
\hline \multicolumn{2}{|l|}{ Tumor grade } \\
\hline Poorly & 100.0 \\
\hline Moderately & 77.5 \\
\hline Well & 0 \\
\hline \multicolumn{2}{|c|}{ Adjuvant therapy } \\
\hline No & 47.4 \\
\hline Yes & 0 \\
\hline \multicolumn{2}{|c|}{ Capsule invasion } \\
\hline No & 0 \\
\hline Yes & 21.2 \\
\hline
\end{tabular}

NP, neutrophil percentage; CA199, carbohydrate antigen 199; $\mathrm{T}$, tumor.

prognostic factors for pancreatic adenocarcinoma $(9-11,14)$. In particular, T4 stage is associated with the presence of tumors involved in the coeliac axis or superior mesenteric artery (28). In addition, since patients with $\mathrm{T} 4$ stage tumors may not be able to undergo surgery, patients with T4 stage pancreatic adenocarcinoma were not included in the study.

Consistent with previous findings, the results of the present study demonstrated that, in addition to the $\mathrm{T}$ and $\mathrm{N}$ stages, tumor grade may also act as an independent predictor for patients with resected pancreatic adenocarcinoma $(14,31,37)$. For example, it has been reported that well-differentiated tumors are significantly associated with longer survival rates $(14,37,38)$. The nomogram developed in the present study demonstrated the magnitude of poor prognosis as tumor grade changed from well to poorly differentiated. It has been reported that tumor grade incorporation into the current TNM system enables accurate prognosis prediction within particular clinical stages (30), which has been validated by two subsequent studies $(11,37)$. In the present study, patients with distinct tumor grades were assigned to different points
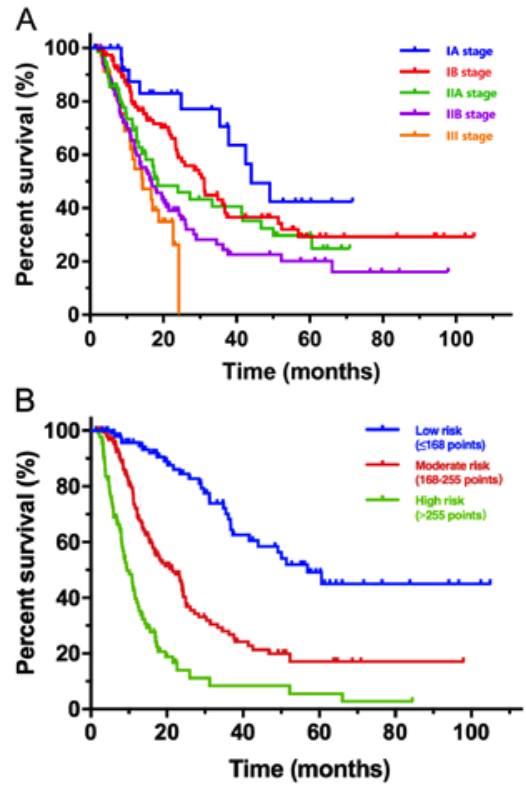

Figure 3. Kaplan-Meier survival curves for patients with resected pancreatic adenocarcinoma, according to (A) the TNM staging system and (B) the risk stratification system based on the developed nomogram. TNM, tumor-node-metastasis.

in the nomogram, even if they were classified within the same TNM stage. These observations partly illustrated the higher power of the nomogram for predicting the survival of patients compared with the TNM staging system.

Consistent with previous findings (39-41), the results from the present study demonstrated that presence of capsule invasion was an independent poor prognostic factor for OS in patients with resected pancreatic adenocarcinoma. In another study, Mannell et al (39) reported that the malignant invasion of the pancreatic capsule was significantly associated with poor prognosis. Furthermore, it has been demonstrated that the incidence of capsule invasion has a tendency to increase in relation to tumor size (40). This phenomenon may explain why capsule invasion is considered a poor prognosis factor.

CA199 is a well-established marker used to determine tumor burden, and the most frequently used tumor marker for pancreatic adenocarcinoma $(14,42,43)$. CA199 has been reported as a diagnostic and a prognostic marker (14-16). Evaluation of preoperative CA199 is positively associated with tumor resectability and postoperative prognosis $(14,16,44,45)$. Furthermore, postoperative CA199 levels have been reported to predict OS and disease-free survival following cancer resection and adjuvant chemotherapy (45-49). Consistent with the results from the present study, a previous report demonstrated that CA199 can predict the prognosis of patients with resected pancreatic adenocarcinoma when combined with PLR (45). A 10 -year follow-up study on a large patient population indicated that CA199 is an independent prognostic factor for advanced pancreatic adenocarcinoma (50).

In the nomogram developed in the present study, blood transfusion was a prognostic factor that could have been easily ignored. Previous studies reported that blood transfusion is associated with the survival outcome of patients following pancreatic resection (51-53). Furthermore, a previous study identified blood transfusion as a significant negative predictor 

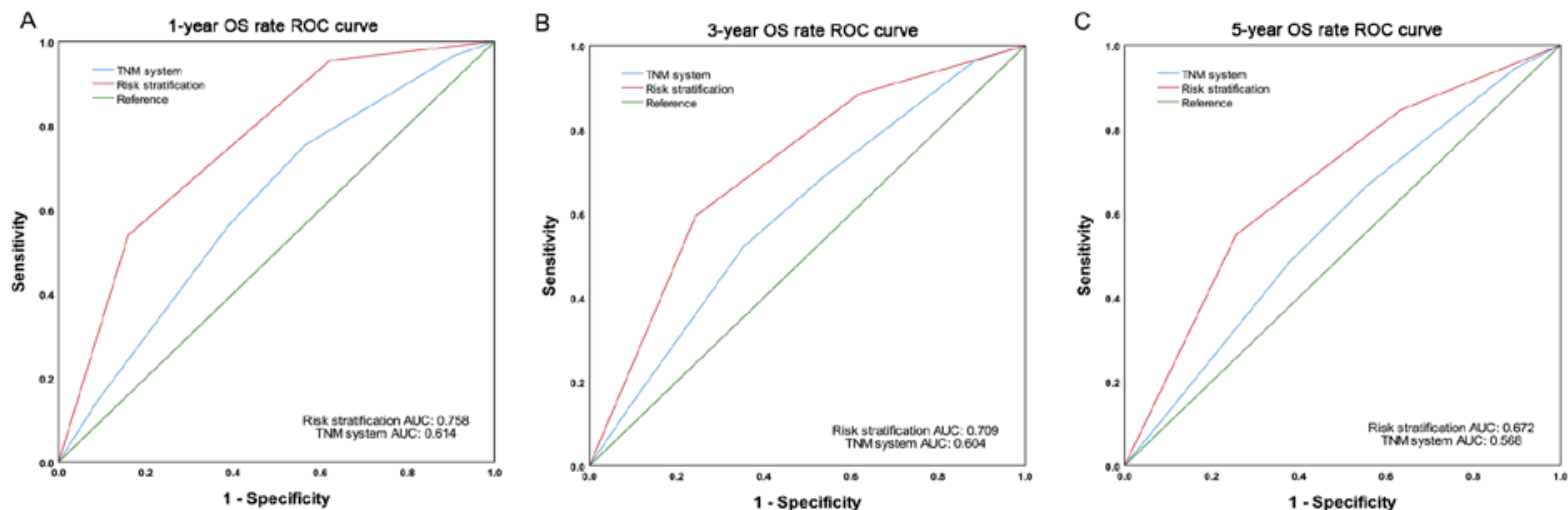

Figure 4. Comparisons of the ROC curves of the risk stratification system, based on the developed nomogram and the TNM staging systems for (A) 1-year, (B) 3-year and (C) 5-year OS prediction. ROC, receiver operating characteristic; TNM, tumor-node-metastasis; OS, overall survival; AUC, area under the curve.

of survival, whereas adjuvant chemotherapy is associated with significantly longer survival (53). These findings are consistent with the results from the present study. Furthermore, intraoperative transfusion, lymph node metastasis and lymph node ratio have been reported as independent prognostic factors in predicting tumor recurrence (54). However, how intraoperative transfusion may have a negative impact on the recurrence and OS of patients with cancer remains unclear. It has been hypothesized that blood transfusion may suppress the immune system of the recipient, resulting therefore in early tumor recurrence (55). Conversely, it has been speculated that patients requiring blood transfusion may have been associated with perioperative complications, which may influence survival more than the transfusion itself (56). Prospective studies are therefore required to determine the clinical impact and underlying mechanism of transfusion in patients with pancreatic adenocarcinoma. However, blood transfusion should be avoided by following the established operative procedures, in order to minimize intraoperative bleeding.

Adjuvant therapy has been reported to be associated with the prognosis of patients following pancreatic adenocarcinoma resection (57-61). Although adjuvant therapy is not a pathological factor, it is considered to significantly affect survival (59-61). It was therefore included in the nomogram developed in the present study. A systematic review reported that despite curative-intent resection, the prognosis of patients with recurrence remains poor, which leads therefore to adjuvant therapy (61). Consistent with the results from the present study, Corsini et al (60) reported that patients with pancreatic adenocarcinoma who receive adjuvant therapy following successful resection have better survival rates. These findings were described in numerous studies (57-59). Subsequently, according to the present study and previous reports, it is suggested that patients with pancreatic adenocarcinoma should undergo postoperative chemotherapy.

It has been demonstrated that NP may act as a prognostic factor for patients with resected pancreatic adenocarcinoma. Previous studies reported the association between inflammation and various types of malignancy, such as renal cell carcinoma, pancreatic cancer (62-65), and cancer-associated inflammation is ranked as the seventh most common hallmark of tumor development (66). Based on systemic inflammation analyses, several prognostic factors, including NLR, MLR and PNI, have the ability to predict the prognosis of patients with resectable pancreatic adenocarcinoma (67-69). Consistent with these findings, the present study identified NLR as a prognostic factor for pancreatic cancer following univariate analysis; however, multivariate analysis failed to validate NLR role as an independent prognostic factor. Furthermore, the association between NP and the prognosis of patients with pancreatic adenocarcinoma has been well established. For example, pretreatment with NP has been reported to act as an independent prognostic factor for patients with advanced cancer who exhibit adverse outcomes (70). NP has also been considered as an independent prognostic factor in patients with nasopharyngeal carcinoma (71). Previous studies suggested that neutrophils, which serve a crucial role in the inflammatory tumor microenvironment, could mediate the pro-tumor effects via different molecular mechanisms $(72,73)$. In addition, neutrophils are associated with increased tumor burden and tumor aggressiveness, which may reflect the prognosis of patients with various types of cancer (70). The results from the present study demonstrated that NP may be considered as a novel prognostic indicator for predicting survival outcome of patients with pancreatic cancer. However, whether NP, as an immune index, may be used as a prognostic marker for patients with resected pancreatic adenocarcinoma requires further investigation using larger-scale cohort studies.

Validation is essential to assure that the nomogram is universally applicable (21). In the present study, the calibration plots in the validation set exhibited a positive association between the predicted nomogram and the actual survival rate, which demonstrated the repeatability and reliability of the present nomogram. Furthermore, the $\mathrm{C}$-indices in both training and validation sets were significantly higher in the nomogram compared with the TNM staging system. In addition, a clear risk stratification system of OS rates was established by using the total scores obtained from the nomogram. In the present study, the AUC values indicated that the risk stratification system based on the nomogram demonstrated improved ability in predicting the 1-year, 3-year and 5-year OS rates compared with the TNM system.

To the best of our knowledge, the present study was the first to describe a nomogram that included tumor marker, immune index, surgical information, pathological data and adjuvant therapy to predict the OS of patients with resected 
pancreatic adenocarcinoma. The present nomogram demonstrated favorable discrimination and calibration in the training and validation sets. It may therefore be used as a practical tool to predict the prognosis of patients.

The present study presented some limitations. Firstly, this study lacked an external validation. In the present study, $70 \%$ of patients were randomly assigned into the training set to develop the nomogram, whereas $30 \%$ of patients were assigned into the validation set to validate the nomogram. Although this is a generally accepted method for nomogram development and validation, external validation based on other populations is required to estimate the model accuracy. Secondly, although the validation results demonstrated that the nomogram may be able to predict the prognosis of patients, this nomogram did not include all potential prognostic factors such as preoperative treatment, familial pancreatic adenocarcinoma, the number of harvested lymph nodes etc. Thirdly, due to the complexity of adjuvant therapy, specific adjuvant therapy options were not subdivided in the present study, which may have influenced the accuracy of the prediction.

In conclusion, the present study successfully developed and validated a nomogram that could be used for the prognosis prediction of patients with resected pancreatic adenocarcinoma, based on the database from the China National Cancer Center. Compared with the TNM staging system, the present nomogram was more performant at predicting prognosis of patients. This nomogram requires further external validation before its use in clinical practice.

\section{Acknowledgements}

Not applicable.

\section{Funding}

The present study was funded by the CAMS Innovation Fund for Medical Sciences (grant no. 2016-I2M-1-001).

\section{Availability of data and materials}

The datasets used and/or analyzed during the present study are available from the corresponding author upon reasonable request.

\section{Authors' contributions}

HR and CFW conceived and designed the present study. HR and CRW drafted the initial manuscript and performed statistical analysis. HR, CRW and SA acquired the data. SA performed the statistical analysis. HR and CFW analyzed and interpreted the data. All authors contributed to data analysis. All authors drafted and critically revised the paper for important intellectual content. All authors read and approved the final manuscript.

\section{Ethics approval and consent to participate}

The present study was approved by the Ethics Committee of National Cancer Center/Cancer Hospital, Chinese Academy of Medical Sciences and Peking Union Medical College (Beijing, China).

\section{Patient consent for publication}

Not applicable.

\section{Competing interests}

The authors declare that they have no competing interests.

\section{References}

1. Chen W, Zheng R, Baade PD, Zhang S, Zeng H, Bray F, Jemal A Yu XQ and He J: Cancer statistics in China, 2015. CA Cancer J Clin 66: 115-132, 2016.

2. Siegel RL, Miller KD and Jemal A: Cancer statistics, 2018. CA Cancer J Clin 68: 7-30, 2018.

3. Rahib L, Smith BD, Aizenberg R, Rosenzweig AB, Fleshman JM and Matrisian LM: Projecting cancer incidence and deaths to 2030: The unexpected burden of thyroid, liver, and pancreas cancers in the United States. Cancer Res 74: 2913-2921, 2014.

4. Kamisawa T, Wood LD, Itoi T and Takaori K: Pancreatic cancer. Lancet 388: 73-85, 2016.

5. Heestand GM, Murphy JD and Lowy AM: Approach to patients with pancreatic cancer without detectable metastases. J Clin Oncol 33: 1770-1778, 2015.

6. Ferrone CR, Brennan MF, Gonen M, Coit DG, Fong Y, Chung S, Tang L, Klimstra D and Allen PJ: Pancreatic adenocarcinoma: The actual 5-year survivors. J Gastrointest Surg 12: 701-706, 2008.

7. Katz MH, Wang H, Fleming JB, Sun CC, Hwang RF, Wolff RA Varadhachary G, Abbruzzese JL, Crane CH, Krishnan S, et al: Long-term survival after multidisciplinary management of resected pancreatic adenocarcinoma. Ann Surg Oncol 16: 836-847, 2009.

8. Cameron JL and He J: Two thousand consecutive pancreaticoduodenectomies. J Am Coll Surg 220: 530-536, 2015.

9. Allen PJ, Kuk D, Castillo CF, et al: Multi-institutional Validation Study of the American Joint Commission on Cancer (8th Edition) Changes for $\mathrm{T}$ and $\mathrm{N}$ Staging in Patients With Pancreatic Adenocarcinoma. Ann Surg 265: 185-191, 2017.

10. Chatterjee D, Katz MH, Foo WC, Sundar M, Wang H, Varadhachary GR, Wolff RA, Lee JE, Maitra A, Fleming JB, et al: Prognostic Significance of New AJCC Tumor Stage in Patients With Pancreatic Ductal Adenocarcinoma Treated With Neoadjuvant Therapy. Am J Surg Pathol 41: 1097-1104, 2017.

11. Rochefort MM, Ankeny JS, Kadera BE, Donald GW, Isacoff W, Wainberg ZA, Hines OJ, Donahue TR, Reber HA and Tomlinson JS: Impact of tumor grade on pancreatic cancer prognosis: Validation of a novel TNMG staging system. Ann Surg Oncol 20: 4322-4329, 2013.

12. Yuan C, Morales-Oyarvide V, Babic A, Clish CB, Kraft P, Bao Y, Qian ZR, Rubinson DA, Ng K, Giovannucci EL, et al: Cigarette Smoking and Pancreatic Cancer Survival. J Clin Oncol 35: 1822-1828, 2017.

13. Ferrone CR, Kattan MW, Tomlinson JS, Thayer SP, Brennan MF and Warshaw AL: Validation of a postresection pancreatic adenocarcinoma nomogram for disease-specific survival. J Clin Oncol 23: 7529-7535, 2005.

14. Barhli A, Cros J, Bartholin L and Neuzillet C: Prognostic stratification of resected pancreatic ductal adenocarcinoma: Past, present, and future. Dig Liver Dis 50: 979-990, 2018.

15. Ballehaninna UK and Chamberlain RS: The clinical utility of serum CA 19-9 in the diagnosis, prognosis and management of pancreatic adenocarcinoma: An evidence based appraisal. J Gastrointest Oncol 3: 105-119, 2012.

16. Humphris JL, Chang DK, Johns AL, Scarlett CJ, Pajic M, Jones MD, Colvin EK, Nagrial A, Chin VT, Chantrill LA, et al; NSW Pancreatic Cancer Network: The prognostic and predictive value of serum CA19.9 in pancreatic cancer. Ann Oncol 23: 1713-1722, 2012

17. Albert JM, Liu DD, Shen Y, Pan IW, Shih YC, Hoffman KE, Buchholz TA, Giordano SH and Smith BD: Nomogram to predict the benefit of radiation for older patients with breast cancer treated with conservative surgery. J Clin Oncol 30: 2837-2843, 2012.

18. Yi M, Meric-Bernstam F, Kuerer HM, Mittendorf EA, Bedrosian I, Lucci A, Hwang RF, Crow JR, Luo S and Hunt KK: Evaluation of a breast cancer nomogram for predicting risk of ipsilateral breast tumor recurrences in patients with ductal carcinoma in situ after local excision. J Clin Oncol 30: 600-607, 2012. 
19. Tendulkar RD, Agrawal S, Gao T, Efstathiou JA, Pisansky TM, Michalski JM, Koontz BF, Hamstra DA, Feng FY, Liauw SL, et al: Contemporary Update of a Multi-Institutional Predictive Nomogram for Salvage Radiotherapy After Radical Prostatectomy. J Clin Oncol 34: 3648-3654, 2016.

20. Nam RK, Kattan MW, Chin JL, Trachtenberg J, Singal R, Rendon R, Klotz LH, Sugar L, Sherman C, Izawa J, et al: Prospective multi-institutional study evaluating the performance of prostate cancer risk calculators. J Clin Oncol 29: 2959-2964, 2011.

21. Iasonos A, Schrag D, Raj GV and Panageas KS: How to build and interpret a nomogram for cancer prognosis. J Clin Oncol 26: 1364-1370, 2008.

22. Zhang ZY, Luo QF, Yin XW, Dai ZL, Basnet S and Ge HY: Nomograms to predict survival after colorectal cancer resection without preoperative therapy. BMC Cancer 16: 658, 2016.

23. Brennan MF, Kattan MW, Klimstra D and Conlon K: Prognostic nomogram for patients undergoing resection for adenocarcinoma of the pancreas. Ann Surg 240: 293-298, 2004.

24. Song W, Miao DL and Chen L: Nomogram for predicting survival in patients with pancreatic cancer. OncoTargets Ther 11: $539-545,2018$.

25. Pu N, Li J, Xu Y, Lee W, Fang Y, Han X, Zhao G, Zhang L, Nuerxiati A, Yin $\mathrm{H}$, et al: Comparison of prognostic prediction between nomogram based on lymph node ratio and AJCC 8th staging system for patients with resected pancreatic head carcinoma: A SEER analysis. Cancer Manag Res 10: 227-238, 2018.

26. He C, Zhang Y, Cai Z, Lin X and Li S: Overall survival and cancer-specific survival in patients with surgically resected pancreatic head adenocarcinoma: A competing risk nomogram analysis. J Cancer 9: 3156-3167, 2018.

27. He C, Zhong L, Zhang Y, Cai Z and Lin X: Development and validation of a nomogram to predict liver metastasis in patients with pancreatic ductal adenocarcinoma: A large cohort study. Cancer Manag Res 11: 3981-3991, 2019.

28. Chun YS, Pawlik TM and Vauthey JN: 8th Edition of the AJCC Cancer Staging Manual: Pancreas and Hepatobiliary Cancers. Ann Surg Oncol 25: 845-847, 2018.

29. Camp RL, Dolled-Filhart M and Rimm DL: X-tile: A new bio-informatics tool for biomarker assessment and outcome-based cut-point optimization. Clin Cancer Res 10: 7252-7259, 2004.

30. Wasif N, Ko CY, Farrell J, Wainberg Z, Hines OJ, Reber H and Tomlinson JS: Impact of tumor grade on prognosis in pancreatic cancer: Should we include grade in AJCC staging? Ann Surg Oncol 17: 2312-2320, 2010.

31. Huang L, Balavarca Y, van der Geest L, Lemmens V, Van Eycken L, De Schutter H, Johannesen TB, Zadnik V, Primic-Žakelj M, Mägi M, et al: Development and validation of a prognostic model to predict the prognosis of patients who underwent chemotherapy and resection of pancreatic adenocarcinoma: A large international population-based cohort study. BMC Med 17: 66, 2019.

32. Diwakarla C, Hannan K, Hein N and Yip D: Advanced pancreatic ductal adenocarcinoma - Complexities of treatment and emerging therapeutic options. World J Gastroenterol 23: 2276-2285, 2017.

33. Fang C, Wang W, Feng X, Sun J, Zhang Y, Zeng Y, Wang J, Chen H, Cai M, Lin J, et al: Nomogram individually predicts the overall survival of patients with gastroenteropancreatic neuroendocrine neoplasms. Br J Cancer 117: 1544-1550, 2017

34. Liang W, Zhang L, Jiang G, Wang Q, Liu L, Liu D, Wang Z, Zhu Z, Deng Q, Xiong X, et al: Development and validation of a nomogram for predicting survival in patients with resected non-small-cell lung cancer. J Clin Oncol 33: 861-869, 2015

35. de Castro SM, Biere SS, Lagarde SM, Busch OR, van Gulik TM and Gouma DJ: Validation of a nomogram for predicting survival after resection for adenocarcinoma of the pancreas. Br J Surg 96 417-423, 2009.

36. He C, Zhang Y, Cai Z, Duan F, Lin X and Li S: Nomogram to Predict Cancer-Specific Survival in Patients with Pancreatic Acinar Cell Carcinoma: A Competing Risk Analysis. J Cancer 9: 4117-4127, 2018.

37. Hlavsa J, Cecka F, Zaruba P, Zajak J, Gurlich R, Strnad R, Pavlik T, Kala $Z$ and Lovecek M: Tumor grade as significant prognostic factor in pancreatic cancer: Validation of a novel TNMG staging system. Neoplasma 65: 637-643, 2018.

38. Garcea G, Dennison AR, Ong SL, et al: Tumour characteristics predictive of survival following resection for ductal adenocarcinoma of the head of pancreas. Eur J Surg Oncol 33: 892-897, 2007.
39. Mannell A, van Heerden JA, Weiland LH and Ilstrup DM: Factors influencing survival after resection for ductal adenocarcinoma of the pancreas. Ann Surg 203: 403-407, 1986.

40. Tsuchiya R, Oribe T and Noda T: Size of the tumor and other factors influencing prognosis of carcinoma of the head of the pancreas. Am J Gastroenterol 80: 459-462, 1985.

41. Nagakawa T, Sanada H, Inagaki M, Sugama J, Ueno K, Konishi I, Ohta T, Kayahara $\mathrm{M}$ and Kitagawa $\mathrm{H}$ : Long-term survivors after resection of carcinoma of the head of the pancreas: Significance of histologically curative resection. J Hepatobiliary Pancreat Surg 11: 402-408, 2004

42. Winter JM, Yeo CJ and Brody JR: Diagnostic, prognostic, and predictive biomarkers in pancreatic cancer. J Surg Oncol 107: 15-22, 2013.

43. Itzkowitz SH, Yuan M, Fukushi Y, Lee H, Shi ZR, Zurawski V Jr, Hakomori S and Kim YS: Immunohistochemical comparison of Lea, monosialosyl Lea (CA 19-9), and disialosyl Lea antigens in human colorectal and pancreatic tissues. Cancer Res 48: $3834-3842,1988$

44. Brown EG, Canter RJ and Bold RJ: Preoperative CA 19-9 kinetics as a prognostic variable in radiographically resectable pancreatic adenocarcinoma. J Surg Oncol 111: 293-298, 2015

45. Sakamoto T, Saito H, Amisaki M, Tokuyasu N, Honjo S and Fujiwara Y: Combined preoperative platelet-to-lymphocyte ratio and serum carbohydrate antigen 19-9 level as a prognostic factor in patients with resected pancreatic cancer. Hepatobiliary Pancreat Dis Int 18: 278-284, 2019.

46. Landi F, Dopazo C, Sapisochin G, Beisani M, Blanco L, Caralt M, Balsells J and Charco R: Long-term results of pancreaticoduodenectomy with superior mesenteric and portal vein resection for ductal adenocarcinoma in the head of the pancreas. Cir Esp 93. 522-529, 2015.

47. Takagi C, Kikuchi Y, Shirakawa H, Hoshimoto S, Tomikawa M, Ozawa I, Hishinuma S and Ogata Y: Predictive Factors for Elevated Postoperative Carbohydrate Antigen 19-9 Levels in Patients With Resected Pancreatic Cancer. Anticancer Res 39: 3177-3183, 2019.

48. Aoki S, Motoi F, Murakami Y, Sho M, Satoi S, Honda G, Uemura K, Okada KI, Matsumoto I, Nagai M, et al; Multicenter Study Group of Pancreatobiliary Surgery (MSG-PBS): Decreased serum carbohydrate antigen 19-9 levels after neoadjuvant therapy predict a better prognosis for patients with pancreatic adenocarcinoma: A multicenter case-control study of 240 patients. BMC Cancer 19: 252, 2019.

49. Imaoka H, Shimizu Y, Senda Y, et al: Post-adjuvant chemotherapy CA19-9 levels predict prognosis in patients with pancreatic ductal adenocarcinoma: A retrospective cohort study. Pancreatology 16: 658-664, 2016.

50. Deng QL, Dong S, Wang L, Zhang CY, Ying HF, Li ZS, Shen XH, Guo YB, Meng ZQ, Yu JM, et al: Development and Validation of a Nomogram for Predicting Survival in Patients with Advanced Pancreatic Ductal Adenocarcinoma. Sci Rep 7: 11524, 2017.

51. Mitra A, Pai E, Dusane R, Ranganathan P, DeSouza A, Goel M and Shrikhande SV: Extended pancreatectomy as defined by the ISGPS: Useful in selected cases of pancreatic cancer but invaluable in other complex pancreatic tumors. Langenbecks Arch Surg 403: 203-212, 2018.

52. Homeyer RS, Roberts KJ, Sutcliffe RP, Kaltenborn A, Mirza D, Qu Z, Klempnauer J and Schrem H: Ventilation after pancreaticoduodenectomy increases perioperative mortality: Identification of risk factors and their relevance in Germany that do not apply in England. Hepatobiliary Pancreat Dis Int 18: 379-388, 2019.

53. Petrou A, Soonawalla Z, Silva MA, et al: Prognostic indicators following curative pancreatoduodenectomy for pancreatic carcinoma: A retrospective multivariate analysis of a single centre experience. J BUON 21: 874-882, 2016.

54. Hwang HK, Jung MJ, Lee SH, Kang CM and Lee WJ: Adverse oncologic effects of intraoperative transfusion during pancreatectomy for left-sided pancreatic cancer: The need for strict transfusion policy. J Hepatobiliary Pancreat Sci 23: 497-507, 2016

55. Hoh H, Umpleby H, Cooper A and Taylor I: Recurrence of colorectal cancer and perioperative blood transfusion. Is blood storage time important? Dis Colon Rectum 33: 127-130, 1990.

56. Park SJ, Kim SW, Jang JY, Lee KU and Park YH: Intraoperative transfusion: Is it a real prognostic factor of periampullary cancer following pancreatoduodenectomy? World J Surg 26: 487-492, 2002.

57. Fitzgerald TL, Hunter L, Mosquera C, Jindal C, Biswas T, Zervos E and Efird JT: A simple matrix to predict treatment success and long-term survival among patients undergoing pancreatectomy. HPB (Oxford) 21: 204-211, 2019. 
58. Ahmad NA, Lewis JD, Ginsberg GG, Haller DG, Morris JB Williams NN, Rosato EF and Kochman ML: Long term survival after pancreatic resection for pancreatic adenocarcinoma. Am J Gastroenterol 96: 2609-2615, 2001.

59. Morganti AG, Falconi M, van Stiphout RG, Mattiucci GC, Alfieri S, Calvo FA, Dubois JB, Fastner G, Herman JM, Maidment BW III, et al: Multi-institutional pooled analysis on adjuvant chemoradiation in pancreatic cancer. Int $\mathrm{J}$ Radiat Oncol Biol Phys 90: 911-917, 2014.

60. Corsini MM, Miller RC, Haddock MG, Donohue JH, Farnell MB Nagorney DM, Jatoi A, McWilliams RR, Kim GP, Bhatia S, et al: Adjuvant radiotherapy and chemotherapy for pancreatic carcinoma: The Mayo Clinic experience (1975-2005). J Clin Oncol 26: 3511-3516, 2008.

61. Yegya-Raman N, Shah MM, Grandhi MS, Poplin E, August DA, Kennedy TJ, Malhotra U, Spencer KR, Carpizo DR and Jabbour SK: Adjuvant therapeutic strategies for resectable pancreatic adenocarcinoma. Ann Pancreat Cancer 1: 1, 2018.

62. Peng D, Li XS, Zhang CJ, et al: Prognostic factors of patients with T3NOM0 renal cell carcinoma: a single-center retrospective study of 182 patients. Beijing Da Xue Xue Bao Yi Xue Ban 48: 806-811, 2016 (In Chinese).

63. Demir IE, Friess H and Ceyhan GO: Neural plasticity in pancreatitis and pancreatic cancer. Nat Rev Gastroenterol Hepatol 12 649-659, 2015

64. Mantovani A, Allavena P, Sica A and Balkwill F: Cancer-related inflammation. Nature 454: 436-444, 2008.

65. Grivennikov SI, Greten FR and Karin M: Immunity, inflammation, and cancer. Cell 140: 883-899, 2010.

66. Hanahan D and Weinberg RA: Hallmarks of cancer: The next generation. Cell 144: 646-674, 2011.
67. Proctor MJ, Morrison DS, Talwar D, Balmer SM, O'Reilly DS, Foulis AK, Horgan PG and McMillan DC: An inflammation-based prognostic score (mGPS) predicts cancer survival independent of tumour site: A Glasgow Inflammation Outcome Study. Br J Cancer 104: 726-734, 2011.

68. Stotz M, Gerger A, Eisner F, Szkandera J, Loibner H, Ress AL, Kornprat P, AlZoughbi W, Seggewies FS, Lackner C, et al: Increased neutrophil-lymphocyte ratio is a poor prognostic factor in patients with primary operable and inoperable pancreatic cancer. Br J Cancer 109: 416-421, 2013.

69. Ye S and Bai L: Comparison and validation of the value of preoperative inflammation marker-based prognostic scores in resectable pancreatic ductal adenocarcinoma. Cancer Manag Res 10: 3405-3417, 2018.

70. Zhao W, Wang P, Jia H, Chen M, Gu X, Liu M, Zhang Z, Cheng W and Wu Z: Neutrophil count and percentage: Potential independent prognostic indicators for advanced cancer patients in a palliative care setting. Oncotarget 8: 64499-64508, 2017.

71. He JR, Shen GP, Ren ZF, Qin H, Cui C, Zhang Y, Zeng YX and Jia WH: Pretreatment levels of peripheral neutrophils and lymphocytes as independent prognostic factors in patients with nasopharyngeal carcinoma. Head Neck 34: 1769-1776, 2012.

72. Powell DR and Huttenlocher A: Neutrophils in the Tumor Microenvironment. Trends Immunol 37: 41-52, 2016.

73. Uribe-Querol E and Rosales C: Neutrophils in Cancer: Two Sides of the Same Coin. J Immunol Res 2015: 983698, 2015.

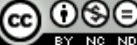

This work is licensed under a Creative Commons
Attribution-NonCommercial-NoDerivatives 4.0 International (CC BY-NC-ND 4.0) License. 